\title{
Optimization-based features extraction for K-complex detection
}

\author{
Z. Roshan Zamir ${ }^{1} \quad$ N. Sukhorukova ${ }^{2} \quad$ H. Amiel $^{3}$ \\ A. Ugon $^{4} \quad$ C. Philippe ${ }^{5}$
}

(Received 17 December 2013; revised 19 August 2014)

\begin{abstract}
The K-complex is a transient electroencephalogram (EEG, brain activity) waveform that contributes to sleep stage scoring. An automated detection of $\mathrm{K}$-complexes is an important component of sleep stage monitoring. This automation is difficult due to the stochastic nature of brain signals, presence of noise, complexity, and extreme size of data. We develop an optimization model, based on solving a sequence of linear least squares problems, to extract key features of EEG signals. The proposed approach significantly reduces the dimension of the problem and the computational time while the classification accuracy is enhanced in most cases. Numerical results show that this procedure is efficient in detecting K-complexes.
\end{abstract}

http://journal.austms.org.au/ojs/index.php/ANZIAMJ/article/view/7802 gives this article, (c) Austral. Mathematical Soc. 2014. Published August 27, 2014, as part of the Proceedings of the 11th Biennial Engineering Mathematics and Applications Conference. ISSN 1446-8735. (Print two pages per sheet of paper.) Copies of this article must not be made otherwise available on the internet; instead link directly to this URL for this article. 


\section{Contents}

1 Introduction

C385

2 Data acquisition

C388

3 Preprocessing and features extraction

C388

3.1 Numerical experiments . . . . . . . . . . . . . . . . C391

3.2 Classifiers . . . . . . . . . . . . . . . C392

4 Results and discussion

C393

5 Conclusion

C394

References

C395

\section{Introduction}

Currently there are an alarming number of people suffering from sleep disorders. The diagnosis of such disorders is performed in a polysomnogram (PSG) test. PSG studies a series of biomedical signals such as brain activity (EEG), muscle movements, heart beat and eye movement. The analysis of EEG, in particular for sleep stage identification, is an active research area in biomedical signal processing.

K-complexes are specific EEG waveforms [6]. They consist of an initial small negative, somewhat sharp wave, followed by a large positive wave. Figure 1 presents a K-complex in an EEG signal, where the amplitude increases sharply and then returns to the original value. Since EEG signals are non-linear, non-stationary and not repeatable, K-complexes have a wide variety of shapes and are difficult to distinguish from other EEG waves. The usual method for sleep stage identification is visual (manual) inspection of an EEG signal by a 


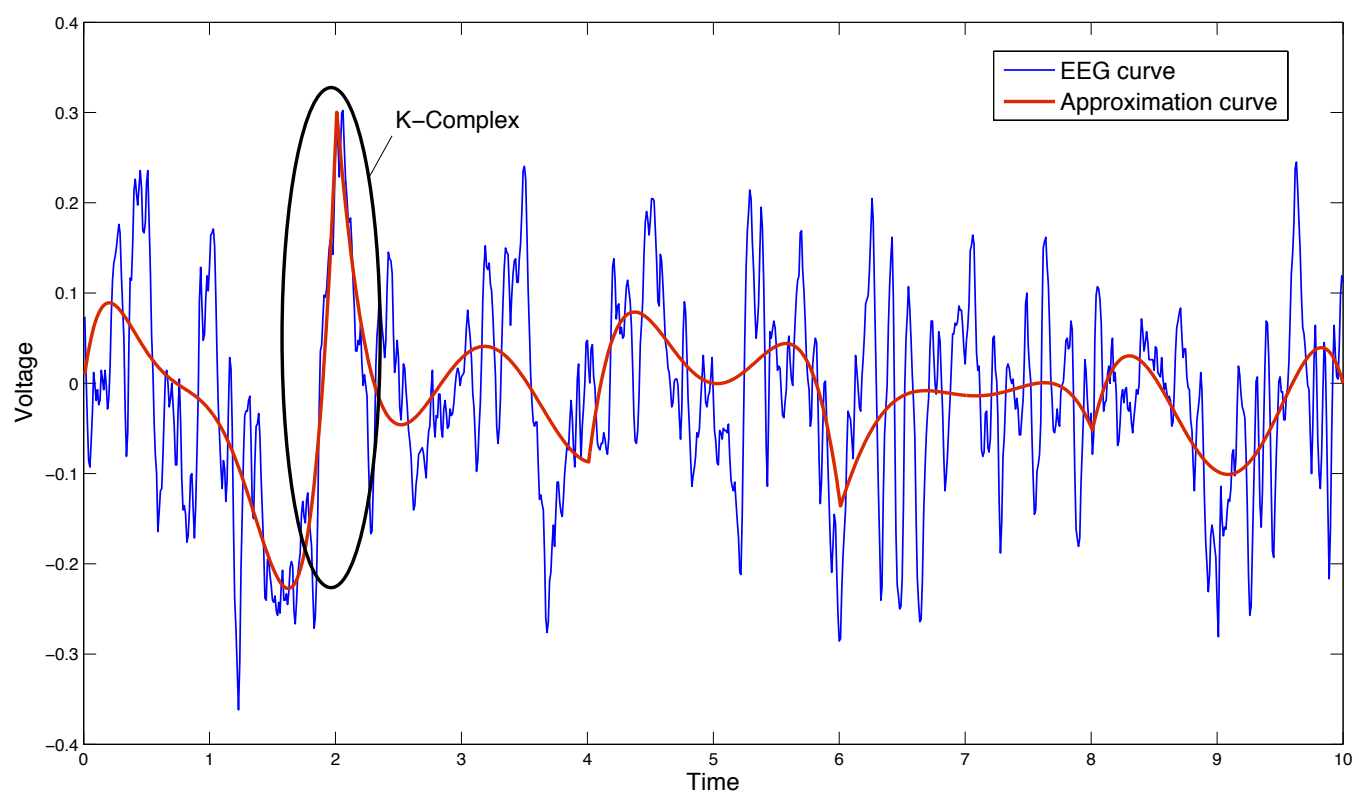

Figure 1: The growth of the amplitude in the presence of K-complexes.

sleep specialist. However, the visual scoring of whole night EEG recordings is a time consuming task.

EEG recordings show that human sleep is categorized into two stages, rapid eye movement (REM) sleep and non-REM (NREM) sleep. Typically, people begin the sleep cycle with a period of NREM sleep followed by a very short period of REM sleep. The American Academy of Sleep Medicine (AASM) divides NREM into three further stages [8].

NREM stage 1 is the transition from waking to sleep.

NREM stage 2 is signaled by K-complexes in the EEG.

NREM stage 3 is called slow-wave-sleep (SwS) or delta sleep. It is the deepest stage of sleep in which the sleeper is least responsive to the environment [8]. 
A patient's sleep cycle begins with NREM stage 1 , followed by NREM stage 2 and then NREM stage 3; after NREM stage 3, the patient returns to NREM stage 2 and then enters REM sleep. Qualified doctors manually analyze the relevant biomedical signals. The sleep stage scoring is based on the standardized scoring Rechtschaffen and Kales (R\&K) rules [10]. K-complexes are defined by this set of rules. One of the major deficiencies of these rules is arbitrarily defined thresholds for sleep stage identification [12]. This can lead to unreliable results and poor agreement between scorers. While a few studies were carried out to automate the detection of K-complexes based on artificial neural networks [9], wavelet transforms [14], matched filters [13] and an electronic system using filters, pulsers, and threshold detectors [5], no standard algorithm is accepted by the medical community. Medical doctors report that the accuracy of K-complex detection is not satisfactory as it remains subjective $[1,8]$. Therefore, an accurate method for automatic detection of K-complexes is very desirable. This automation would reduce the number of manual tasks significantly, thereby making the process more reliable and cost efficient.

We propose a new algorithm based on an optimization model for the automatic detection of K-complexes. This consists of two major steps. First, we extract essential features from a single EEG signal and reduce the dimensionality of the data. Our feature extraction procedure is based on optimization. Second, we apply classification algorithms to evaluate the accuracy of EEG classifications (K-complex detection).

The main contribution of this work is the development of the feature extraction procedure, which is based on a careful choice of the class of functions that model the amplitude efficiently. These functions have two important properties. First, they approximate the shape of the actual amplitude functions. Second, the corresponding optimization problems are able to be solved accurately and inexpensively. Section 3 presents our proposed procedure for $\mathrm{K}$-complex detection as preprocessing and a feature extraction procedure. We approximate the brain signal (EEG) by a sinusoidal curve (approximate wave or model wave) with the amplitude approximated by a piecewise polynomial 
function. Then we minimize the sum of the squares of the deviations between the original signal and the fitted wave (modeling function). After extracting the essential features, classification algorithms are applied over the obtained set of features to enhance their classification accuracy. Results of numerical experiments are presented in Section 4.

\section{Data acquisition}

There are several difficulties in the development of efficient methods for $\mathrm{K}$-complex detection. One difficulty is data availability since the scoring of K-complexes is not normally kept in PSG analysis records. Therefore, we requested that medical practitioners prepare the data for this research. Another difficulty is that the same segment of data may be scored differently by different scorers [8]. In our experiments, we use data scored at Tenon Hospital in Paris.

In this study each observation contains a 10 second segment of an EEG recording at a sampling frequency of $100 \mathrm{~Hz}$. Each data segment consists of a sequence of $\left(t_{i}, y_{i}\right), i=1,2, \ldots, 1000$, where $y_{i}$ is the EEG voltage recorded at time $t_{i}$. A dataset with 39 non-K-complexes and $31 \mathrm{~K}$-complexes (70 observations) is used.

\section{Preprocessing and features extraction}

Many signals are modeled as a sine wave $A(t) \sin [\omega(t) t+\tau(t)]$, where $A(t)$ is the amplitude, $\omega(t)$ is the frequency and $\tau(t)$ is the shift (phase). The choice of these three functions is important. Consider

$$
\min _{A, \omega, \tau} \sum_{i=1}^{N}\left\{y_{i}-A(t) \sin [\omega(t) t+\tau(t)]\right\}^{2}
$$


where $y_{i}, i=1, \ldots, N$ are the EEG recordings at time $t_{i}$, with $N$ the total number of recordings. For modeling any kind of signal it is reasonable to consider all $A(t), \omega(t)$ and $\tau(t)$ as functions of time $t$; however, the corresponding optimization problem may become too complex. Therefore, our goal is to find a suitable equilibrium, where the corresponding functions are:

- precise enough to describe the signal and produce high accuracy classification; and

- simple enough to be solved accurately and inexpensively on a PC used in a medical lab.

Since one of the main characteristics of K-complexes is a sudden increase in signal amplitude, we model the amplitude as a piecewise polynomial function (spline function). Spline functions are suitable to describe such abrupt amplitude changes and therefore it is very natural to use them in amplitude modeling. Section 3.1 shows that the corresponding optimization problems are relatively inexpensive to solve.

There are many ways to construct polynomial splines. One possibility is through a truncated power function:

$$
S_{m}(x, \theta, t)=x_{0}+\sum_{j=1}^{m} x_{1 j} t^{j}+\sum_{l=2}^{n} \sum_{j=1}^{m} x_{l j}\left(t-\theta_{l-1}\right)_{+}^{j},
$$

where $m$ is the degree, $n$ is the number of intervals in a 10 seconds duration of an EEG, $\theta=\left(\theta_{1}, \ldots, \theta_{n-1}\right)$ are the knots, $x_{0}, x_{11}, \ldots, x_{n m}$ are the spline parameters and

$$
\left(t-\theta_{l-1}\right)_{+}=\max \left[0,\left(t-\theta_{l-1}\right)\right]= \begin{cases}t-\theta_{l-1} & \text { if } t>\theta_{l-1}, \\ 0 & \text { if } t \leqslant \theta_{l-1},\end{cases}
$$

is the truncated power function. The spline knots can be free or fixed. If the knots are chosen to be free, then they are additional variables in the 
optimization problem. Thus, $\boldsymbol{x}=\left(x_{0}, x_{11}, \ldots, x_{n m}, \boldsymbol{\theta}\right)$ and the corresponding optimization problem becomes more complex; moreover, it becomes nonconvex. Generally, it is much easier to solve a higher dimension fixed knots problem than a free knots problem. To specify the amplitude approximated by a polynomial spline, its parameters and knots (if they are free) must be determined.

Assume that the range of possible frequencies and phases form a fine grid which is precise enough for the purposes of this problem and not very large (details are in Section 3.1). In this case, for each possible combination of $\omega$ and $\tau$ values, the only function we need to approximate is the amplitude. The amplitude is modeled as a polynomial spline whose knots are fixed and the parameters are to be optimized. Then, the optimization problem is

$$
\min _{x} \sum_{i=1}^{N}\left\{y_{i}-S_{m}\left(x, \theta, t_{i}\right) \sin \left(\omega t_{i}+\tau\right)\right\}^{2} .
$$

This is a linear least squares problem and therefore the solution of the original optimization problem (1) is reduced to solving a sequence of linear least squares problems (LLSPS) when $\omega$ and $\tau$ are constants (on the grid, and we keep the best combination).

Equation (3) is rewritten as

$$
\min _{x} \sum_{i=1}^{N}\left(y_{i}-M x\right)^{2} \text { or } \min _{x}\left\|M x-y_{i}\right\|_{2}^{2},
$$

where $y_{i}$ are the recorded signals, $x \in \mathbb{R}^{m n+1}$ and $M$ is a matrix with $m n+1$ columns and $N$ rows of the form $S_{m}\left(\theta, t_{i}\right) \sin \left(\omega t_{i}+\tau\right)$. If $M \in$ $\mathbb{R}^{N \times(m n+1)}$ is a full rank matrix, then the LLSP is solved through the system of normal equations. There exist various methods for solving LLSP based on normal equations [4], QR decomposition [15] and singular value decomposition (SVD) [3]. Solving the system of normal equations $\left(M^{\top} M\right) x=M^{\top} b$ is the most common method when the matrix $M^{\top} M$ is known to be nonsingular and well conditioned [2]. 


\subsection{Numerical experiments}

In our experiments we model the amplitude function as a spline function of degree four $(m=4)$ with fixed $\operatorname{knots}(n=5)$ :

$$
S_{4}=x_{0}+x_{11} t+x_{12} t^{2}+x_{13} t^{3}+x_{14} t^{4}+\sum_{l=2}^{5} \sum_{j=1}^{4} x_{l j}\left(t-\theta_{l-1}\right)_{+}^{j},
$$

with the vector optimization variable

$$
x=\left(x_{0}, x_{11}, x_{12}, x_{13}, x_{14}, x_{21}, x_{22}, x_{23}, x_{24}, \ldots, x_{51}, x_{52}, x_{53}, x_{54}\right) .
$$

In all our experiments $M$ is a full-rank matrix. It is possible to show that for this particular application and dataset this will always be the case, although we will not present the proof here. The proof relies on the K-complex almost never lasting less than 1 second, and thus $n \leqslant 10, m \leqslant 5$ and $\omega=20 \mathrm{~Hz}$ are demonstrated through the direct study of the structure of matrix $M$. Therefore, our experiments $(n=5$ and $m=4)$ did not encounter any singularity.

The knots were chosen to be equidistant. The frequency grid was specified between $0.1 \mathrm{~Hz}$ and $15 \mathrm{~Hz}$ with the step size of $1 \mathrm{~Hz}$ (for EEG analysis, medical doctors almost never consider frequencies above $20 \mathrm{~Hz}$ ). Interval $[0, \pi]$ with the step size $\pi / 4$ was assigned to $\tau$.

In the case of $S_{4}$, the dimension of $x$ is $21(m n+1=21, m=4, n=5)$. Also, three more parameters which characterize the improvement of the objective function after linear least squares optimization are considered. These three parameters are the value of the objective function, $\omega$ and $\tau$. Therefore, $\mathrm{N}=1000$ features of the original data are reduced to 24 essential features. 


\subsection{Classifiers}

We used 12 classifiers (classification algorithms) from Weka [16] (open source data analysis software). We only provide a very short description of the classifiers used in this study, more detail is provided by Weka [16].

oner a classifier that uses the minimum error attribute for prediction, discretizing numeric attributes.

REPTree a fast decision tree learner. It builds a decision/regression tree using information gain/variance and prunes it using reduced-error pruning.

LMT a logistic model tree based approach, with logistic regression functions at the leaves.

LibSVM integrated software for support vector machine (SVM) classification.

SMo a sequential minimal optimization algorithm for training a support vector classifier (a special case of LibsvM).

J48 a classifier based on a $\mathrm{C} 4.5^{1}$ decision tree.

RBF a classifier that implements a normalized Gaussian radial basis function network, using the K-means clustering algorithm to provide the basis functions.

KStar an instance-based classifier.

LWL a locally weighted learning classifier that uses an instance based algorithm to assign instance weights.

LazyIBK a K-nearest neighbors classifier.

Logistic a generalized linear model used for binomial regression.

All these classifiers were used with their default sets of parameters except LazyIBK, which was used with $K=1,5$. As a training set we used a dataset

${ }^{1} \mathrm{C} 4.5$ is an algorithm in Weka to generate a decision tree or it is a decision tree classifier. 
with 30 non-K-complexes and $21 \mathrm{~K}$-complexes (51 observations) and a dataset with 9 non-K-complexes and $10 \mathrm{~K}$-complexes (19 observations) was used as a test set. Each of the 12 classifiers were trained on the training set and tested on the test set. The accuracy on the test set is reported.

\section{Results and discussion}

Figure 1 illustrates the approximation curve and original EEG signal. The approximation amplitude is considerably larger at the K-complex. Although the approximation does not follow precisely the trend of original data, it is sufficient to detect the K-complex and therefore to produce the correct classification results. The computational time to extract key features of the EEG is roughly 21 seconds on a PC with $3.10 \mathrm{GHz} \mathrm{CPU}$ and $8 \mathrm{~GB}$ of memory.

First, the classifiers were used over the original dataset with 1000 features. Then all the above classifiers were applied to the obtained set of features (obtained after linear least squares preprocessing). All classification accuracy results are presented in Table 1. It is seen that Logistic does not produce any result on the original dataset. This is most probably due to the large size of the data (1000 features). The accuracy of all classifiers except LibSVM and RBF was considerably improved by using the data with extracted features rather than the original dataset. Although the RBF classifier provides better accuracy on the original dataset than after preprocessing, no classification method failed on the preprocessed data (the set of features obtained through LLSP). The most accurate classifiers were Logistic, LMT and J48 with an accuracy of $74 \%$. The accuracy of most of the classification methods from Weka [16] were considerably improved after LLSP. Our experiments show that LLSP is an appropriate and successful feature extractor for classifying a $\mathrm{K}$-complex in an EEG. 
Table 1: Classification accuracy on the test set for (a) the original dataset; and (b) the preprocessed dataset.

\begin{tabular}{lcc} 
Classifier & Test set accuracy (a) & Test set accuracy (b) \\
\hline OneR & $37 \%$ & $47 \%$ \\
REPTree & $37 \%$ & $47 \%$ \\
LMT & $42 \%$ & $74 \%$ \\
LibSVM & $47 \%$ & $47 \%$ \\
SMO & $47 \%$ & $63 \%$ \\
J48 & $47 \%$ & $74 \%$ \\
RBF & $74 \%$ & $53 \%$ \\
KStar & $47 \%$ & $58 \%$ \\
LWL & $47 \%$ & $53 \%$ \\
LazyIB5 & $53 \%$ & $58 \%$ \\
LazyIB1 & $53 \%$ & $63 \%$ \\
Logistic & $\mathrm{N} / \mathrm{A}$ & $74 \%$
\end{tabular}

\section{Conclusion}

A new optimization-based procedure for detecting K-complexes in an EEG signal is proposed. The proposed procedure works well on the available data, but the shortage of freely available datasets prevents us from testing our method on larger datasets and comparing with other researchers' approaches. Our new procedure has two advantages. Firstly, LLSP-based preprocessing allows one to reduce the size of a classification problem and extract the key features of an EEG signal. Secondly, the accuracy of all of the classification algorithms from Weka [16] except LibSVM and RBF were considerably improved after the preprocessing.

Although our optimization based approach performed well, there are several avenues for future work. One of the main problems is the singularity of matrix $M$. Due to round-off errors [7], matrix $M$ might be close to singular. In this case, rigorous techniques, in particular, QR decomposition and singular 
value decomposition (SVD) can be investigated to get more accurate results. In future work we will also provide a formal study on the values of the parameters $(n, m, w)$, when the corresponding matrix $M$ is full-rank. Another important direction is to develop more efficient ways to obtain these parameters, for example, a Fourier transform in combination with a 'branch and cut'-based procedure [11].

Acknowledgments This work is supported by Swinburne University of Technology and is partially supported by CSIRO/ANZIAM Student Support Scheme.

\section{References}

[1] R. Agarwal and J. Gotman. Digital tools in polysomnography. J. Clin. Neurophysiol., 19(2):136-143, 2002.

http://journals. Iww.com/clinicalneurophys/Abstract/2002/ 03000/Digital_Tools_in_Polysomnography.4.aspx. C387

[2] J. L. Barlow. Numerical aspects of solving linear least squares problems. Technical report, Computer Science Department, The Pennsylvania State University, University Park, PA, USA, January 1999. www.cse.psu.edu/ barlow/book.ps. C390

[3] A. Björck. Numerical Methods for Least Squares Problems. Handbook of Numerical Analysis. SIAM, 1996. doi:10.1137/1.9781611971484. C390

[4] S. Boyd and L. Vandenberghe. Convex Optimization. Cambridge University Press, New York, NY, USA, 2010. http://www. cambridge. org/au/academic/subjects/statistics-probability/ optimization-or-and-risk/convex-optimization?format=HB. C390 
[5] G. Bremer, J. R. Smith, and I. Karacan. Automatic detection of the K-complex in sleep electroencephalograms. IEEE T. Bio-Med. Eng., 17(4):314-323, 1970. doi:10.1109/TBME.1970.4502759. C387

[6] P. R. Carney, R. B. Berry, and J. D. Geyer. Clinical Sleep Disorders. LWW medical book collection. Lippincott Williams and Wilkins, 2005. http://www. Iww. com/Product/9780781786928. C385

[7] G. H. Golub and C. F. Van Loan. Matrix Computations. Johns Hopkins Studies in the Mathematical Sciences. Johns Hopkins University Press, Baltimore, MD, USA, 1996.

http://portal.acm.org/citation. cfm?id=248979. C394

[8] C. Iber, S. Ancoli-Israel, A. L. Chesson, and S. F. Quani. AASM manual for the scoring of sleep and associated events. Rules, technology and technical specifications. Technical report, AASM, Westchester, IL, 2007. http://www . aasmnet.org/scoringmanual/v2.0.2/html/ index.html? IXDevelopmentProcess.html. C386, C387, C388

[9] B. H. Jansen. Artificial neural nets for K-complex detection. IEEE Eng. Med. Biol., 9(3):50-52, 1990. doi:10.1109/51.59213. C387

[10] A. Kales, A. Rechtschaffen, Los Angeles University of California, and NINDB Neurological Information Network (U.S.). A manual of standardized terminology, techniques and scoring system for sleep stages of human subjects. U. S. National Institute of Neurological Diseases and Blindness, Neurological Information Network Bethesda, Md, 1968. http://nla.gov.au/nla.cat-vn823711. C387

[11] J. E. Mitchell. Branch-and-cut algorithms for combinatorial optimization problems. In P. M. Pardalos and M. G. C. Resende, editors, Handbook of Applied Optimization, pages 65-77. Oxford University Press, 2002. http://global.oup.com/academic/product/ handbook-of-applied-optimization- 9780195125948 ? cc=au\&lang= en\&. C395 
[12] D. Moloney, N. Sukhorukova, P. Vamplew, J. Ugon, G. Li, G. Beliakov, C. Philippe, H. Amiel, and A. Ugon. Detecting K-complexes for sleep stage identification using nonsmooth optimization. ANZIAM J., 52:319-332, 2011. doi:10.1017/S1446181112000016. C387

[13] O. Sheriff, B. Pagnrek, S. Mamouhd, and R. Broughton. Automatic detection of K-complex in sleep EEG. Int. Electrical Electronic Conf. Exp., 81, 1977. C387

[14] Z. Tang and N. Ishii. Detection of the K-complex using a new method of recognizing waveform based on the discrete wavelet transform. IEICE T. Inf. Syst., E78-D(1):77-85, 1995.

http://search.ieice.org/bin/summary.php?id=e78-d_1_77. C387

[15] L. N. Trefethen and D. Bau. Numerical Linear Algebra. SIAM, 1997. http://bookstore.siam.org/ot50/. C390

[16] Weka web site. www.cs.waikato.ac.nz/ml/weka/. C392, C393, C394

\section{Author addresses}

1. Z. Roshan Zamir, Faculty of Engineering and Industrial Sciences, Swinburne University of Technology, PO Box 218, Hawthorn, Victoria, Australia. mailto:zroshanzamir@swin.edu.au

2. N. Sukhorukova, Faculty of Engineering and Industrial Sciences, Swinburne University of Technology, PO Box 218, Hawthorn, Victoria, Australia. mailto:nsukhorukova@swin.edu.au

3. H. Amiel, Hôpital Tenon 4, rue de la Chine 75970 Paris Cedex 20 France. mailto:amielhelene@gmail.com 
4. A. Ugon, Hôpital Tenon 4, rue de la Chine 75970 Paris Cedex 20 France.

mailto:adrien.ugon@tnn.aphp. fr

5. C. Philippe, Hôpital Tenon 4, rue de la Chine 75970 Paris Cedex 20 France.

mailto:carole.philippe@tnn.aphp.fr 